\title{
Erratum to: Semigroups of partial isometries
}

\author{
Alexey I. Popov • Heydar Radjavi
}

Received: 17 June 2013 / Accepted: 17 June 2013

C Springer Science+Business Media New York 2013

\section{Erratum to: Semigroup Forum \\ DOI 10.1007/s00233-013-9487-6}

One of the corollaries to the first main result of our paper is not true as it stands: Theorem 3.9 and its proof should be replaced as follows.

Theorem Let $\mathcal{S}$ be a self-adjoint finitely generated semigroup of partial isometries. That is, $\mathcal{S}=\left\langle T_{1}, T_{1}^{*}, \ldots, T_{n}, T_{n}^{*}\right\rangle$ for some $T_{1}, \ldots, T_{n}$. If $\operatorname{ran} T_{i}=\operatorname{ran} T_{j}, \operatorname{ker} T_{i}=$ $\operatorname{ker} T_{j}$ and $T_{i} T_{j}=T_{j} T_{i}$ for all $i$ and $j$, then $\mathcal{S}$ is atomic in the sense that the measure space from Theorem 3.1 corresponding to $\mathcal{S}$ is purely atomic.

Proof For each $i$, let $\mathcal{L}_{i}$ be the lattice of subspaces of $\mathcal{H}$ generated by the ranges and the kernels of $T_{i}^{k}, k=0,1,2, \ldots$. Let us show that $\mathcal{L}_{i}=\mathcal{L}_{j}$ for all $i$ and $j$. It is enough to establish that $\operatorname{ran} T_{i}^{k}=\operatorname{ran} T_{j}^{k}$ and $\operatorname{ker} T_{i}^{k}=\operatorname{ker} T_{j}^{k}, k=0,1,2, \ldots$ If $k=0$, the statement is trivial. Assume by induction that the statement is proved for some $k$. Pick $x \in \operatorname{ran} T_{i}^{k+1}$. There is $y \in \mathcal{H}$ such that $x=T_{i}^{k+1} y=T_{i} T_{i}^{k} y$. Since $\operatorname{ran} T_{i}^{k}=\operatorname{ran} T_{j}^{k}$, there is $z \in \mathcal{H}$ such that $T_{i}^{k} y=T_{j}^{k} z$. Then $x=T_{i} T_{j}^{k} z$. By commutativity, $x=T_{j}^{k} T_{i} z$. Since $\operatorname{ran} T_{i}=\operatorname{ran} T_{j}$ by the assumptions of the theorem, there is $v \in \mathcal{H}$ such that $x=T_{j}^{k} T_{j} v=T_{j}^{k+1} v$. So, $\operatorname{ran} T_{i}^{k+1}=\operatorname{ran} T_{j}^{k+1}$. Similarly,

Communicated by Norman R. Reilly.

The online version of the original article can be found under doi:10.1007/s00233-013-9487-6.

A.I. Popov $\cdot$ H. Radjavi (凶)

Department of Pure Mathematics, University of Waterloo, Waterloo, ON N2L 3G1, Canada e-mail: hradjavi@uwaterloo.ca

A.I. Popov

e-mail: a4popov@uwaterloo.ca 
if $x \in \operatorname{ker} T_{i}^{k+1}$, that is, $T_{i}^{k+1} x=0$, then $T_{i}\left(T_{i}^{k} x\right)=0$. Assuming by induction that $\operatorname{ker} T_{i}^{k}=\operatorname{ker} T_{j}^{k}$, we get $T_{i}\left(T_{j}^{k} x\right)=0$. By commutativity, $T_{j}^{k}\left(T_{i} x\right)=0$. Again, using the assumptions of the theorem, $T_{j}^{k}\left(T_{j} x\right)=T_{j}^{k+1} x=0$. So, $\operatorname{ker} T_{i}^{k+1}=\operatorname{ker} T_{j}^{k+1}$. This shows that $\mathcal{L}_{i}=\mathcal{L}_{j}$ for all $i$ and $j$.

For each $i=1, \ldots, n$, let $\mathcal{S}_{i}$ denote the singly generated semigroup $\left\langle T_{i}, T_{i}^{*}\right\rangle$. By Theorem 2.1, each $\mathcal{S}_{i}$ is atomic (in the sense of Theorem 3.1). It readily follows from $\mathcal{L}_{i}=\mathcal{L}_{j}$ that the atoms corresponding to $\mathcal{S}_{i}$ are the same as those for $\mathcal{S}_{j}(i, j=$ $1, \ldots, n)$. So, each generator (and, hence, each member) of $\mathcal{S}$, when applied to any of these atoms, turns it either into zero or into another atom.

Remark There are examples showing that without the range assumption or the kernel assumption the statement is not true even with commuting generators.

There is also a more obvious omission of the word "irreducible" in the statement of Corollary 3.12. 